\title{
Revolution durch Reden: Emanzipatorische Kommunikationswissenschaft und Diskursverweigerung passen nicht zusammen
}

\section{Zu Rudolf Stöbers Beitrag ,Genderstern und Binnen-I. Zu falscher Symbolpolitik in Zeiten eines zunehmenden Illiberalismus“ (Publizistik, Heft 1, 2021) und dem Offenen Brief gegen dessen Veröffentlichung}

\section{Uwe Krüger}

Angenommen: 27. Februar 2021 / Online publiziert: 17. März 2021

(C) Der/die Autor(en) 2021

\section{Vorbemerkung}

Als in die Jahre kommender weißer, heterosexueller Cis-Mann, überdies auf einer Dauerstelle im akademischen Mittelbau, bin ich in vielfacher Hinsicht privilegiert. Ich habe nie Sexismus, Homophobie oder Rassismus am eigenen Leib erfahren; als ostdeutsches Arbeiterkind habe ich zwar einige habitusbezogene Karriere-Handicaps, doch bin ich nicht mehr abhängig vom Wohlwollen der Mitglieder von Berufungs- oder Besetzungskommissionen. Diese kumulierten Privilegien haben mit Sicherheit einen Einfluss darauf, wie ich die aktuelle Debatte um Rudolf Stöbers Artikel „Genderstern und Binnen-I“ (Stöber 2020) wahrnehme - so wie die sozialen Positionen anderer Akteur*innen (privilegierten wie unterprivilegierten) deren Wahrnehmungen und Sprechakte prägen dürften.

Ich bin weiterhin nicht auf der Höhe der akademischen Debatten rund um diskriminierungsfreie Sprache, ich habe zu Gendertheorie oder feministischen Themen weder gelehrt noch geforscht. Ich bin in diesem Sinne Laie und interessierter Beobachter von Konflikten rund um dieses Thema. Als Mitglied im Organisationsteam des Netzwerks Kritische Kommunikationswissenschaft (KriKoWi) sehe ich mich aber als Teil und Unterstützer emanzipatorischer Bewegungen, die sich gegen Diskriminierungen in Sachen class, race und gender richten (vgl. van den Ecker und Tröger 2021). Als Mitherausgeber von KriKoWi-Sammelbänden redigiere ich Autor*innen (ja, auch weiblichen) den Gender-Stern in die Manuskripte hinein; ich verwende Sonderzeichen wie den Stern oder den Doppelpunkt in E-Mails und achte

Dr. U. Krüger $(\bowtie)$

Institut für Kommunikations- und Medienwissenschaft, Lehr- und Forschungsbereich Journalismus, Universität Leipzig, Nikolaistr. 27-29, 04109 Leipzig, Deutschland

E-Mail: uwe.krueger@uni-leipzig.de 
in meinen Lehrveranstaltungen auf genderneutrale oder inklusive Formulierungen in meinen Sätzen.

Ich bin dabei kein Vorkämpfer, sondern habe eine Achtsamkeit für dieses Thema antrainiert bekommen - von meinen KriKoWi-Kolleg*innen, von unseren Studierenden, die heute fast alle den Gender-Gap mitsprechen (anders als noch vor zwei, drei Jahren) und vom allgemeinen öffentlichen Diskurs, vom medialen Mainstream. Ich fühle mich dabei nicht manipuliert oder zensiert, sondern sehe die zunehmende Rücksichtnahme auf marginalisierte Gruppen als zivilisatorischen Fortschritt an, den ich sehr gern mitgehe - weil ich überzeugt bin, dass sprachliche Repräsentation einen realen Unterschied in Emanzipationsfragen machen oder diesen zumindest vorbereiten kann. Diesen Wertewandel, diese „moralische Revolution“ (vgl. Appiah 2011) in Sachen Antidiskriminierung trage ich gern mit.

Dementsprechend überzeugt mich der Beitrag Stöbers inhaltlich in kaum einer Hinsicht. (Es gibt nur einen Punkt, in dem ich ihm voll zustimme - dazu später.) Dennoch finde ich die Veröffentlichung seines Beitrags durch die Publizistik richtig und den Offenen Brief an den DGPuK-Vorstand ${ }^{1}$ als Reaktion darauf falsch. Im Folgenden möchte ich dies begründen und hoffe, mit manchen Überlegungen eine Perspektive für einen Weg aus dem Kommunikationsinfarkt aufzuzeigen.

\section{Zum Artikel ,Genderstern und Binnen-I““}

Anders als die über 350 Fachkolleg*innen, die mit dem Offenen Brief gegen die Veröffentlichung des Beitrags protestiert haben und ihn offenbar vor allem aufgrund von als diffamierend empfundenen Reizwörtern unerträglich finden, meine ich, dass man sich mit der Position von Rudolf Stöber auseinandersetzen kann. Ich referiere zunächst seine aus meiner Sicht wichtigsten Argumente, ohne diese Reizwörter in den Mittelpunkt zu stellen:

1. Stöber wendet sich offenbar nicht gegen gendergerechte Sprache an sich, sondern nur gegen Genderstern und Binnen-I. Diese seien „derzeit sprachnormwidrig“ (1), als wichtigste Instanzen führt Stöber den zwischenstaatlichen „Rat für deutsche Rechtschreibung“ und mit Abstrichen den Duden an. „Und gegen andere Formen der ,gendergerechten Sprache “ wende ich mich keineswegs.“ (S. 5)

2. Hinter der Sprachnormverletzung stehe „das Bemühen, die Emanzipation voranzutreiben“ (S. 3) - doch wer ,,bewusst sprachpolitisch eingreift, um via Sprache Denken zu lenken, manipuliert“ (S. 4). Dies sei gefährlich und weise eine Nähe zu totalitären Systemen auf, die ebenfalls „Gedankenmanipulation via Sprachlenkung“ betrieben hätten und „Menschen zu ihrem Glück zwingen“ wollten - so etwas ende ,in der Regel mit der Aufrichtung von Guillotinen“ (ebd.). Es sei der „heraufdämmernde, wachsende Illiberalismus“, den er „fürchte“ (S. 3).

3. Zudem führten Gender-Stern und Binnen-I vermutlich nicht zu realer, ,wünschenswerter Emanzipation“ und dazu, ,reale Diskriminierungen [...] aus der Welt

\footnotetext{
1 http://dgpuk-genderstern.de/.
} 
zu schaffen“, sondern lediglich zur Beschädigung der „Institutionen Schrift und Sprache“ und zur „Polarisierung der Gesellschaft“ (S. 5).

4. Stöber fordert verstärkte Forschung zu der Frage „ob Gender-* und Binnen-I bei den Rezipienten so ankommen, wie sie wirken sollen“. „Man sollte empirisch prüfen, wer auf generische Pluralbildung und wer auf Gender-Stern und Binnen-I empfindlich reagiert.“ (S. 8). Er vermutet, ,dass das Gendern die Mehrzahl der Bevölkerung kaum interessiert“" und es ,außerhalb bestimmter Milieus eher auf Ablehnung stoßen wird“" (S. 9).

Ich bin, wie gesagt, kein Geschlechterforscher und auch kein Sprachforscher, allerdings an Fragen von Ideologie, Manipulation, Framing und Medienmisstrauen (Stichwort „Lügenpresse“) sehr interessiert. Vor diesem Hintergrund möchte ich Stöbers Argumentationsgang, der nicht immer klar und nachvollziehbar ist, wie folgt kritisieren:

Der zentrale Vorwurf, den Stöber den Verfechter*innen der gendergerechten Sprache macht, ist der der „Manipulation“ und der ,Ideologie“ (zwölfmal kommt „,manipul“ im Text vor, neunmal ,ideolog“) - doch diese Begriffe bestimmt er nicht näher. Hätte er dies (auch für sich) geklärt, hätte dies möglicherweise zu einer stringenteren Argumentation geführt. Ich nehme an, er hängt (trotz einer Marx-Referenz an anderer Stelle) nicht dem Marxschen Verständnis von Ideologie als ,notwendig falschem Bewusstsein“ im Kapitalismus an, sondern neigt eher dem Verständnis von Karl Popper zu: Dieser kennzeichnet ,politische Ideologien wie den Faschismus oder Stalinismus durch ihren totalitären Charakter, der grundlegend wahrheitsverleugnend, mythenbildend und diskriminierend gegenüber konkurrierenden Vorstellungen ist" (Amlinger 2018, S. 183). Eine solche explizite Begriffsdefinition aber wirft Fragen auf, die Stöber nicht beantwortet: Welche Wahrheiten werden verleugnet, welche Mythen werden gebildet, welche konkurrierenden Vorstellungen werden wie genau diskriminiert? So bleibt Stöbers Assoziationskette vom Ideologie-Vorwurf bis zur „Guillotine“ reichlich vage. Auffällig ist übrigens, dass ihm beim Thema gefährliche Ideologien ausschließlich Kommunismus und Faschismus bzw. Nationalsozialismus einfallen. Stöber vergisst, dass auch die dritte bedeutende politische Ideologie des 19. und 20. Jahrhunderts, der Liberalismus bzw. Neoliberalismus, in Sachen Menschheitsbeglückung mithilfe von personeller und struktureller Gewalt sowie ideologisierter Diskurse und Begriffe nicht unbeleckt ist - man denke an die neoliberalen „Schock-Therapien“ in Krisenländern und die Zusammenarbeit entsprechender Wirtschaftsideologen mit Folterdiktaturen und Besatzungsmächten (vgl. Klein 2009) sowie an organisierte Think-Tank-Kampagnen zur Beeinflussung des öffentlichen Diskurses, um freie Märkte zu naturalisieren und die Privateigentumsordnung, Sozialstaatsabbau, Privatisierungen und Deregulierungen mit individueller Freiheit zu verschweißen (vgl. Walpen 2004; Chamayou 2019).

Auch eine Klärung von Stöbers Manipulationsbegriff wäre mehr als ein akademisches Glasperlenspiel. Der Begriff „Manipulation“ (französisch etwa „Handhabung“ oder „Bearbeitung“) bezeichnet laut Elsen (2008, S. 447) allgemein „das Beeinflussen des Verhaltens eines Gegenübers zum Nutzen des Manipulators“, und zwar unabhängig davon, ob dies bewusst oder unbewusst geschieht und ob die Beeinflussung erkannt wird oder nicht. Daneben gibt es ,,auch die enge Auffassung von 
Manipulation als Lenkung durch bewusste Beeinflussung, die nicht erkannt wird“ (ebd., S. 448). Nun wäre es spannend, ob Stöber den „Sprach- und Gedankenmanipulateure[n]" (S. 4) verdeckte sprachpolitische Operationen vorwirft, ob sie also die Gesellschaft heimlich ,indoktrinieren“ (S. 4) oder mit offenem Visier, transparent und herrschaftsfrei-diskursiv agieren. Ich selbst fühle mich jedenfalls nicht indoktriniert; ich habe eher den Eindruck, dass mit gendersensibler Sprache nicht Manipulation, sondern Edukation betrieben wird, also ,die gezielte Beeinflussung zum Nutzen des Gegenübers“ (Elsen 2008, S. 447). Mit einer solchen Begriffsexegese taucht die Frage auf, wem gendergerechte Sprache eigentlich nützt: nur den bislang sprachlich unterrepräsentierten Frauen und queeren Menschen (nach dem Motto: deren Gewinn ist der Verlust der bislang Privilegierten) oder vielleicht der gesamten Gesellschaft - wenn nämlich Emanzipation dazu führt, dass bislang unterdrückte oder marginalisierte Gruppen sich frei entfalten können und deren Tatkraft und Kreativität am Ende alle bereichert. Dies scheint mir eine Frage der Wahrnehmung zu sein, die in verschiedenen Milieus ganz unterschiedlich beantwortet wird und die im öffentlichen Diskurs stärker diskutiert werden könnte.

Weiterhin verstehe ich nicht, ab wann für Stöber die Manipulation der Sprache eigentlich beginnt und ob der Sündenfall für ihn tatsächlich erst bei GenderStern und Binnen-I passiert und nicht doch schon vorher, nämlich bei jeglicher Form gendersensibler Sprache, die aus einer Reflexion über sprachliche Repräsentationsverhältnisse resultiert. Stöber bekennt einerseits, dass er „für die historisch gewachsene Sprache plädiert“ (S. 6), und verteidigt es, dass wir ,nicht über jedes Wort nach[denken], das wir sagen“ (S. 4). Andererseits schreibt er, offenbar aus Rücksicht auf Frauen, von „Kolleginnen und Kollegen“ (S. 4) und „Rezipientinnen und Rezipienten“ (S. 8). Böser Verdacht: Manipuliert Stöber hier selbst? Trifft ihn der eigene Vorwurf?² Oder ist Manipulation einfach ein ungeeigneter Analysebegriff, wenn es um das Anliegen geht, vor dem Sprechen und Schreiben einmal nachzudenken? Zudem: Als Historiker, der ,für die historisch gewachsene Sprache plädiert“, müsste er sich auch vorstellen können, dass er zurzeit vielleicht einen historischen Wachstumsprozess der Sprache miterlebt, in dem sich unter vorübergehenden Sprachnormverletzungen neue Normen etablieren, die ein Historiker in hundert Jahren als Teil der historisch gewachsenen Sprache ansehen wird.

Beinahe rührend finde ich auch, wie Stöber von Wording und Framing spricht: „Die Diskussion um Wording und Framing anlässlich des ARD-Gutachtens von Elisabeth Wehling [...] zeigt, dass wir längst auf der schiefen Ebene der Manipulation stehen und wo die Sprache zu rutschen beginnt" (S. 4). Framing und Wording als schlimme Techniken der Manipulation - als ob Stöber nicht selbst auch Rahmen aufspannen und Begriffe mit bestimmten Konnotationen verwenden würde (und ich natürlich auch). Framing ist ein notwendiger und unauslöschlicher Bestandteil aller sprachlichen Kommunikation - das hätte Stöber bei Wehling (2016) nachlesen können oder auf netzpolitik.org, wo Wehlings ARD-Gutachten veröffentlicht wurde (vgl. Beckedahl und Dobusch 2019).

\footnotetext{
2 Auf ähnliche Weise hat die FAZ Stöbers Klage über einen ,allgemeine[n] Sprach-, Bildungs- und Kenntnisverfall“" (S. 7) gegen ihn selbst gewendet, da er ein Zitat von Bertolt Brecht in einen falschen Zusammenhang gestellt hat (vgl. Bahners 2021).
} 
Dies wäre meine Entgegnung - als Laie auf dem Gebiet des eigentlichen Themas. Welch Steilvorlage müsste der Text dann erst sein für eine Replik aus berufenem Munde, von feministischen Kommunikationswissenschaftler*innen und Geschlechterforscher*innen! Welch dankbare Gelegenheit, hier diskursiv einzusteigen, Stöbers Argumente zu entkräften und die eigene sprach-, ideologie- und herrschaftskritische Perspektive zu entfalten! Das ultimative Manifest mit den stärksten Argumenten für gendergerechte Sprache (auch solche, die die Normen des Rates für deutsche Rechtschreibung verletzen mag): Ich hätte es gern in der Publizistik gelesen.

\section{Zum Offenen Brief gegen die Veröffentlichung}

Ich habe Stöbers Artikel als sachlich-rational verhandelbaren, zumutbaren Text nacherzählt. Einige emotionalisierende Begriffe daraus habe ich bisher nicht genannt, so „Marotte“ (S. 8), „Manierismen“ (S. 8), „Gedankenpolizei“ (S. 3), „Neusprech“ (George Orwell)“ (S. 5) ,selbstgleichschaltend“ (S. 4) oder „Goebbels, Hitler, Rosenberg“ (S. 4) - ja, alle diese Wörter kommen in dem gut achtseitigen Beitrag auch vor. Durch die entkontextualisierte Aneinanderreihung aller Reizwörter im Offenen Brief wird der Text dort aber als unfassbare Monstrosität konstruiert, über deren Veröffentlichung man sich nur empören kann.

Mit der Publizistik wird hart ins Gericht gegangen: Es liege ein „Versagen wissenschaftlicher Qualitätssicherung und redaktioneller Verantwortung“ vor; es sei „nicht nachvollziehbar, dass dieser Beitrag in der Publizistik veröffentlicht wurde“; er ,beschädigt die Zeitschrift und das Fach Kommunikationswissenschaft“”. Es habe eine Aufforderung der Zeitschrift an Kolleg*innen gegeben, eine Replik für die Folgeausgabe zu schreiben; ,viele haben sich jedoch dagegen entschieden, weil Anfeindungen und Diffamierungen keine Basis für eine sachliche und wissenschaftlich fundierte Auseinandersetzung sein können“.

Wohlgemerkt: Der Brief richtet sich nicht an Herausgeber*innen oder Redaktion der Zeitschrift, sondern an den Vorstand der Fachgesellschaft, denn die Zeitschrift werde „,durch Mitgliedsbeiträge der DGPuK finanziell unterstützt“ und der Vorstand solle ,eine Debatte über die Verantwortung der Publizistik im Rahmen einer Mitgliederversammlung [...] initiieren“. Während Stöber seine Furcht vor „Illiberalismus“ ausgedrückt hat (die die Verfasser*innen des Offenen Briefes leider bestätigen), äuBern die Autor*innen des Offenen Briefs den Wunsch nach „Fachzeitschriften, in denen das Bemühen um Gerechtigkeit (auch, aber längst nicht nur in der Sprache) nicht diskreditiert wird“. Dies erinnert an den klassischen Konflikt zwischen Freiheit und Sicherheit - aus dem Brief spricht hier das Bedürfnis nach der Sicherheit, nicht diffamiert und verletzt zu werden durch Autor*innen, die ihre Redefreiheit (aus Sicht der Briefunterzeichner*innen) über die Maßen ausreizen.

Bei aller Sympathie für den Wert der „Diversität“ und Solidarität im Kampf gegen „Ungleichheiten und Exklusionen“ (Offener Brief) neige ich hier allerdings der Seite der Freiheit zu. Als Journalist und Journalismusforscher spüre ich ein tiefes Unbehagen dabei, wenn der Konflikt zwischen verletzten Wissenschaftler*innen und einer unabhängigen Zeitschrift zu einer Instanz hin eskaliert wird, die in der Lage wäre, die Zeitschrift finanziell unter Druck zu setzen. Dies erinnert an Versuche, 
redaktionelle Unabhängigkeit und (innere) Pressefreiheit auszuhöhlen, die sonst zuweilen Werbekunden oder Medieneigentümer*innen unternehmen. Meine Solidarität gilt hier den Herausgeber*innen der Publizistik, deren ,Stellungnahme zum Offenen Brief $^{* 3}$ ich absolut nachvollziehbar finde. ${ }^{4}$

Die Qualität von Meinungsbeiträgen zur Selbstverständnisdebatte des Fachs sollte im Diskurs bestimmt werden, nicht per Vor- und Selbstzensur, sondern per Replik und Duplik. Zur Replik war die DGPuK-Fachgruppe Medien, Öffentlichkeit und Geschlecht eingeladen worden. Doch hier hat man sich entschieden, das Schachspiel, das Rudolf Stöber (zugegeben: aggressiv) eröffnet hat, nicht mitzuspielen, sondern das Schachbrett umzuwerfen, einen Versuch des „Agenda Cutting“ zu machen und die Redlichkeit des Publikationsmediums in Zweifel zu ziehen.

Ich habe übrigens den Eindruck, dass eine Art Hostile-Media-Effekt vorliegt. Bekanntlich ist die Wahrnehmung, ein Medium sei der eigenen Position gegenüber nicht wohlgesonnen, umso stärker, je stärker man in ein kontroverses Thema involviert ist und je stärker man das Publikum für empfänglich hält. Zu einer solchen Wahrnehmung neigen wir vermutlich aus dem Grund, ,weil anzunehmen ist, dass ein jeweiliges ,neutrales‘ Publikum ,negativ` beeinflusst wird bzw. die Stellung gegnerischer Gruppen verbessert wird“ (Dohle und Hartmann 2008, S. 25). Im OnlineForum zum Thema unterstellen zwei Mitglieder der Fachgruppe Medien, Öffentlichkeit und Geschlecht der Publizistik, sie würde bewusst und gezielt Aufmerksamkeit und Erregungskaskaden erzeugen und bewirtschaften und dafür die bewährten Themen der Geschlechterforschung nutzen (warum sollte sie das tun?). „Empörung als Kalkül?" nennen sie ihren Beitrag ${ }^{5}$ - müssen sich aber umgekehrt fragen lassen, ob die ganze Empörung vielleicht gar nicht entstanden wäre, wenn Stöbers Artikel mit einer sachkundigen Replik beantwortet worden wäre und nicht mit einem empörten Offenen Brief.

Ein anderes, führendes Fachgruppenmitglied kritisiert angesichts Stöbers ,inhaltlicher Leerstellen und fragwürdiger Sprachbilder“, es handele sich ,nicht um einen Debatten-, sondern um einen Debattenverweigerungsbeitrag, der nicht geeignet ist, den Raum eines kontroversen und produktiven Diskurses zu eröffnen“.6 Aus meiner Sicht eine bemerkenswerte Verkehrung des Sachverhalts. Und: Den Beitrag einfach als ,,indiskutabel“ (ebd.) hinzustellen, widerspricht den Prinzipien intellektueller Redlichkeit in der Wissenschaft. Dazu gehört laut Jaster und Keil (2021), „das Gegenüber wohlwollend zu interpretieren“ und „dem Gesagten den jeweils vernünftigsten Sinn abzugewinnen“.

\footnotetext{
3 https://www.dgpuk.de/sites/default/files/Stellungnahme_Publizistik_zum_Offenen\%20Brief.pdf.

4 Ich würde lediglich kritisch nachfragen, ob ein solcher polemischer Meinungsbeitrag mit mangelnder argumentativer Stringenz und begrifflicher Klarheit auch angenommen worden wäre, wenn er nicht von einem reputierten Professor, sondern von einer jungen Doktorandin eingereicht worden wäre - inwieweit also Statushöhere mit viel Sozialkapital im Fach strukturelle Vorteile beim Zugang zum „Forum“ der Publizistik genießen.

5 https://www.dgpuk.de/de/emp\%C3\%B6rung-als-kalk\%C3\%BCl.html.

${ }^{6}$ https://www.dgpuk.de/de/eine-debatte-gerne-aber-unter-anderen-voraussetzungen.html.
} 


\section{Zum Verhältnis emanzipatorischer Kommunikationswissenschaft zur Öffentlichkeit}

Ich erkenne den Schmerz an, den die Erfahrungen von Sexismus und/oder Homophobie vielen Kolleg*innen zugefügt haben, und patriarchale Strukturen im Wissenschaftssystem haben akademische Karrieren von Feminist*innen erschwert oder sogar verhindert (vgl. Nieberding 2020). Ich nehme auch an, dass die harsche Reaktion auf Stöbers Artikel nicht nur an einem schlichten Genervtsein liegt, altbekannte Argumente schon wieder und immer wieder diskutieren zu müssen, sondern auch in einer Angst, dass bereits erreichte Diskursstände in Sachen Geschlechtergerechtigkeit durch provokante Philippiken gefährdet werden könnten - eben weil sie Zweifelnde und Laien beeindrucken und beeinflussen könnten. Doch zivilisatorische Fortschritte kann man nicht per Tribunal, sondern nur durch fortlaufende Überzeugungsarbeit in einem möglichst herrschaftsfreien Diskurs absichern. Auch die Furcht Stöbers vor Illiberalismus, die er dankenswerterweise klar als solche benannt hat, muss anerkannt werden. Denn wenn sich angsterfüllte Parteien gegenüberstehen, die ihre Position jeweils als prekär wahrnehmen, wird es gefährlich: „Fear leads to anger, anger leads to hate, hate leads to suffering", sagte einmal ein weltbekannter spiritueller Lehrer. ${ }^{7}$

Die Kritik aus der Fachgruppe, dass Stöber sich geäußert habe, ohne sich eingehend mit dem Forschungsstand zur geschlechtergerechten Sprache beschäftigt zu haben (sprich: ohne die 36 Titel gelesen zu haben, die zwei Fachgruppen im Nachhinein zusammengestellt haben ${ }^{8}$ ), mag in der Sache zutreffen. Doch der Anspruch ist für eine offene Gesellschaft sehr hoch geschraubt: Will man im Diskurs (und sei es auch nur im akademischen) ausschließlich „Eingeweihte“ zulassen, die sich durch einen von Expert*innen erstellten Kanon gearbeitet haben? Auch Perspektiven und Fragen von der Seite, mit anderer Literatur im Hintergrund, können wertvoll sein - und wenn sie es nicht sind, können sie mit Wissen aus dem Kanon gekontert werden. Die Meinungs-, Presse- und Wissenschaftsfreiheit ist seinerzeit vom männlichen, weißen Bürgertum dem Adel und der Kirche abgerungen worden, also auch Ergebnis einer emanzipatorischen Bewegung. Sie war und ist auch in den neuen, dringend notwendigen Runden emanzipatorischer Kämpfe essenziell für marginalisierte Gruppen, um sich Gehör zu verschaffen, und sollte nun nicht geopfert werden.

Im Grunde kann man den Beitrag von Stöber als „Nicht-Eingeweihtem“ auch als Einbruch der Wirklichkeit in den Fachdiskurs sehen, als gesellschaftliches Symptom: Sein Wert liegt nicht in einer besonders luziden Argumentation, sondern darin, dass er ein in Teilen der Bevölkerung durchaus verbreitetes Unbehagen ${ }^{9}$ artikuliert und in die akademische Blase hineinträgt, in welcher diskriminierungsfreie Sprache

\footnotetext{
7 https://www.youtube.com/watch?v=91_G8iaokk8.

8 https://www.dgpuk.de/sites/default/files/Literaturliste_Forschungsstand_GGS.pdf.

9 Als Belege für die Verbreitung ablehnender Reaktionen in der Bevölkerung seien ein Zapp-Beitrag zum Thema (Girschick 2021), Umfragen (etwa Börsenblatt 2019) sowie die Beliebtheit von Hashtags wie \#Genderwahn, \#Gendergaga und \#Genderideologie auf Social-Media-Plattformen angeführt. Aufregung über „diesen rechthaberischen, autoritären, arroganten und dazu unästhetischen Unsinn“ findet sich selbst auf der Leserbriefseite der taz (20./21.02.2021, S. 37).
} 
bereits zum Mainstream gehört. Damit eröffnet er die Chance, dieses Unbehagen auf hohem Niveau zu verhandeln, dank Open Access vor potenziell der gesamten deutschsprachigen Öffentlichkeit. Stöber spricht für viele, die mit ihren Steuern auch die Lehrstühle und Stellen von Unterzeichner*innen des Offenen Briefs finanzieren. Denn in einem hat Stöber auf jeden Fall recht: Gendern polarisiert. Das ist so, weil Rücksichtnahmen auf bislang unterprivilegierte Gruppen den bislang Privilegierten etwas abverlangen, weil letztere etwas abgeben müssen an Diskursmacht und Unbedarftheit im Sprechen und Schreiben, und weil Gewohnheiten herausgefordert werden. Weil es aber polarisiert, muss sachlich darüber diskutiert werden. Vielleicht noch jahrzehntelang. Auch wenn es wehtut. Auch wenn es nervt. Zumindest von weißen Inhaber*innen ordentlicher Universitätsprofessuren (Achtung: Privilegien) darf man daher erwarten, dass sie sich mit einer gewissen Toleranz gegenüber Nichtwissen, mit Geduld, Gelassenheit, Vertrauen und dickem Fell den Auseinandersetzungen stellen, die zur Vollendung der Revolution eben noch geführt werden müssen.

Danksagung Das Manuskript wurde im Organisationsteam des Netzwerks Kritische Kommunikationswissenschaft (aus dem einige Mitglieder den Offenen Brief unterzeichnet hatten) kontrovers diskutiert. Ich danke Nils S. Borchers, Yuvviki Dioh, Melanie Malczok, Aljoscha Paulus, Kerem Schamberger, Sebastian Sevignani und Mandy Tröger für ihre Perspektiven und Anregungen (einige davon habe ich berücksichtigt, andere nicht). Weiterhin danke ich Markus Beiler und Holger Pötzsch für ihre Kommentare und Hinweise.

Funding Open Access funding enabled and organized by Projekt DEAL.

Open Access Dieser Artikel wird unter der Creative Commons Namensnennung 4.0 International Lizenz veröffentlicht, welche die Nutzung, Vervielfältigung, Bearbeitung, Verbreitung und Wiedergabe in jeglichem Medium und Format erlaubt, sofern Sie den/die ursprünglichen Autor(en) und die Quelle ordnungsgemäß nennen, einen Link zur Creative Commons Lizenz beifügen und angeben, ob Änderungen vorgenommen wurden.

Die in diesem Artikel enthaltenen Bilder und sonstiges Drittmaterial unterliegen ebenfalls der genannten Creative Commons Lizenz, sofern sich aus der Abbildungslegende nichts anderes ergibt. Sofern das betreffende Material nicht unter der genannten Creative Commons Lizenz steht und die betreffende Handlung nicht nach gesetzlichen Vorschriften erlaubt ist, ist für die oben aufgeführten Weiterverwendungen des Materials die Einwilligung des jeweiligen Rechteinhabers einzuholen.

Weitere Details zur Lizenz entnehmen Sie bitte der Lizenzinformation auf http://creativecommons.org/ licenses/by/4.0/deed.de.

\section{Literatur}

Amlinger, C. (2018). Ideologie. In J. Kopp \& A. Steinbach (Hrsg.), Grundbegriffe der Soziologie (S. 181-184). Wiesbaden: Springer VS. https://doi.org/10.1007/978-3-658-20978-0_37.

Appiah, K. A. (2011). Eine Frage der Ehre oder Wie es zu moralischen Revolutionen kommt. München: C.H. Beck.

Bahners, P. (2021). Schlechte Zeit für Lyrik. Im akademischen Kulturkrieg stirbt die Philologie: Rudolf Stöber schickt Brechts „Kinderhymne“ in den Kampf gegen die Gendersprache. Frankfurter Allgemeine Zeitung vom 17.2. https://zeitung.faz.net/faz/geisteswissenschaften/2021-02-17/schlechtezeit-fuer-lyrik/572837.html. Zugegriffen: 26 Feb 2021.

Beckedahl, M., \& Dobusch, L. (2019). Wir veröffentlichen das Framing-Gutachten der ARD. Netzpolitik.org vom 17.2. https://netzpolitik.org/2019/wir-veroeffentlichen-das-framing-gutachten-der-ard/. Zugegriffen: 26 Feb 2021. 
Börsenblatt (2019). Umfrage zur gendergerechten Sprache: Keine Mehrheit für „BürgerInnenmeister“. Börsenblatt vom 5.4. https://www.boersenblatt.net/archiv/1635567.html. Zugegriffen: 26 Feb 2021.>

Chamayou, G. (2019). Die unregierbare Gesellschaft. Eine Genealogie des autoritären Liberalismus. Berlin: Suhrkamp.

Dohle, M., \& Hartmann, T. (2008). Alles eine Frage hoher Reichweite? Eine experimentelle Untersuchung zur Ursache der Entstehung von Hostile-Media-Effekten. Medien \& Kommunikationswissenschaft, $56,21-41$.

Elsen, H. (2008). Manipulation aus sprachlicher Sicht - ein Überblick. Wirkendes Wort. Deutsche Sprache und Literatur in Forschung und Lehre, 58, 447-466.

Girschick, K. (2021). Gendern - machen oder lassen? Zapp, NDR Fernsehen vom 17.2. https://www. ardmediathek.de/ard/video/zapp/gendern-machen-oder-lassen/ndr-fernsehen/Y3JpZDovL25kci5kZS 80NjU2NzMxYS0zYTEyLTQ0N2UtODczZC1kMmYyZDYxYzMyYWE/. Zugegriffen: 26 Feb 2021.

Jaster, R., \& Keil, G. (2021). Wer an der Uni sprechen darf - und wie. Eine Handreichung für intellektuelle Redlichkeit im Streit um die Wissenschaftsfreiheit. Die Zeit Nr. 7 vom 11.2., S. 34. https://www. zeit.de/2021/07/wissenschaftsfreiheit-universitaet-redefreiheit-rassismus-liberalismus-diskurs. Zugegriffen: 26 Feb 2021.

Klein, N. (2009). Die Schock-Strategie. Der Aufstieg des Katastrophen-Kapitalismus. Frankfurt am Main: Fischer.

Nieberding, M. (2020). „Unsere Grammatik widerspricht dem Grundgesetz“. Interview mit der Linguistin Luise F. Pusch. Süddeutsche Zeitung Magazin Nr. 52 vom 23.12., S. 26-32.

Stöber, R. (2020). Genderstern und Binnen-I. Zu falscher Symbolpolitik in Zeiten eines zunehmenden Illiberalismus. Publizistik. https://doi.org/10.1007/s11616-020-00625-0.

van den Ecker, M., \& Tröger, M. (2021). Zum Kritikbegriff des Netzwerks Kritische Kommunikationswissenschaft. Studies in Communication and Media, 10(2), . Themenheft: Kritik an, in und durch Kommunikations- und Medienwissenschaft (in Vorb.). https://www.scm.nomos.de/

Walpen, B. (2004). Die offenen Feinde und ihre Gesellschaft. Eine hegemonietheoretische Studie zur Mont Pèlerin Society. Hamburg: VSA.

Wehling, E. (2016). Politisches Framing: Wie eine Nation sich ihr Denken einredet und daraus Politik macht. Köln: Herbert von Halem.

Dr. Uwe Krüger Dipl.-Journ., ist Wissenschaftlicher Mitarbeiter am Institut für Kommunikations- und Medienwissenschaft der Universität Leipzig. 